\title{
Time-To-Collision Models for Single-File Pedestrian Motion
}

\author{
Jakob Cordes ${ }^{1,3}$. Mohcine Chraibi ${ }^{1}$. Antoine Tordeux ${ }^{2}$. \\ Andreas Schadschneider ${ }^{3}$ \\ ${ }^{1}$ Civil Safety Research, Forschungszentrum Jülich, Jülich, Germany \\ E-mail: j.cordes@fz-juelich.de,m.chraibi@fz-juelich.de \\ ${ }^{2}$ Institute for Security Technology, University of Wuppertal, Wuppertal, Germany \\ E-mail: tordeux@uni-wuppertal.de \\ ${ }^{3}$ Institute for Theoretical Physics, University of Cologne, Cologne, Germany \\ E-mail: as@thp.uni-koeln.de
}

Received: 25 August 2021 / Last revision received: 11 November 2021 / Accepted: 11 November 2021

DOI: $10.17815 / \mathrm{CD} .2021 .133$

\begin{abstract}
We apply the concept of time-to-collision (TTC) to the modeling of pedestrian dynamics. The TTC combines the spatial distances with the velocities to quantify the 'distance' to a collision. Therefore, it is a promising candidate for modeling the interactions between pedestrians. Empirical studies also indicate that the interaction between pedestrians can be described by the TTC: While the pair distribution of the distances, i.e. the probability of two pedestrians to have a certain spatial distance, was found to strongly depend on the relative velocity, the TTC accurately parametrizes its pair distribution. However, there are still few pedestrian models that use the TTC. After giving a general definition of the TTC, we present the widely used approximations for its calculation, especially in a one-dimensional setting. Combined with a desired time-gap, these give rise to different models, namely an Optimal-Velocity model and a new Time-to-Collision model. The TTC model exhibits, however, generic inconsistencies which are related to the estimates we use to approximate the speed of the predecessor. The estimates have a large impact on the dynamics and must therefore be interpreted as reflecting the pedestrians behavior, i.e. as anticipation strategies. We propose new estimates for the predecessor's speed. These give rise to a rich family of models based on the TTC which are analyzed by means of linear stability analysis and simulations.
\end{abstract}

Keywords Pedestrian dynamics · optimal velocity models · fundamental diagram . single-file motion · time-to-collision 


\section{Introduction}

The behavior of humans in a crowd is generally complex and gives rise to numerous (collective) phenomena. Several key principles of the individual behavior are widely agreed on. Pedestrians occupy a certain volume or area. They actively try to achieve their goals and usually avoid collisions by keeping a safety distance. Furthermore, unlike granular systems, pedestrians anticipate the change of their surroundings [1].

There are many ways to incorporate these features in a microscopic model. Crowds may be described in terms of (social) forces [2], cellular automata [3] or by an optimal velocity which is chosen according to the surroundings [4,5]. In many models, the interaction is based on the spatial distances only. However, the pair distribution of the distances, i.e. the probability of two pedestrians to have a certain spatial distance, calculated from a large collection of data, was found to strongly depend on the relative velocity while the time-to-collision accurately parametrizes its pair distribution [6].

The time-to-collision combines the spatial distances with the (relative) velocities to quantify the 'distance' to a collision. Therefore, it is a promising parameter to be included in pedestrian models [6-9]. However, none of the previous models use the TTC in an Optimal-Velocity-like model. In [10] the underlying problems of diverging times-tocollision and unrealistic high flows are briefly discussed.

In this contribution, it is investigated how the TTC can be used as a fundamental quantity in (minimal) pedestrian models. This could help to understand the way pedestrians (inter-)act and might allow better planning in future.

At first, the time-to-collision is defined and calculated using different approximations. These give rise to different models, the well-known Optimal-Velocity models and a new class of Time-to-Collision models. Fundamental problems of these are discussed and related to the implicitly assumed anticipation strategies. More realistic anticipation schemes are therefore proposed. Some numerical results are shown in the last section.

\section{Time-to-Collision}

Assume two pedestrians $i$ and $j$ which are represented by discs of diameter $l$ as in Fig. 1 . Their spatial distance is defined as

$$
D(t)=\operatorname{dist}(i, j)=\left|\vec{r}_{j}(t)-\vec{r}_{i}(t)\right|
$$

A collision will occur if any time $t_{C} \in[t, \infty)$ exists such that $D\left(t_{C}\right)=l$. The time-tocollision $\tau$ between $i$ and $j$ at the time $t$ is then defined as

$$
\tau^{i j}(t):=t_{C}-t
$$

If no time $t_{C}$ exists, the TTC is set to infinity. In the case of multiple times $t_{C}$, their minimum determines the time-to-collision.

Since the future evolution is usually not known, the TTC is typically calculated using the following approximations: 


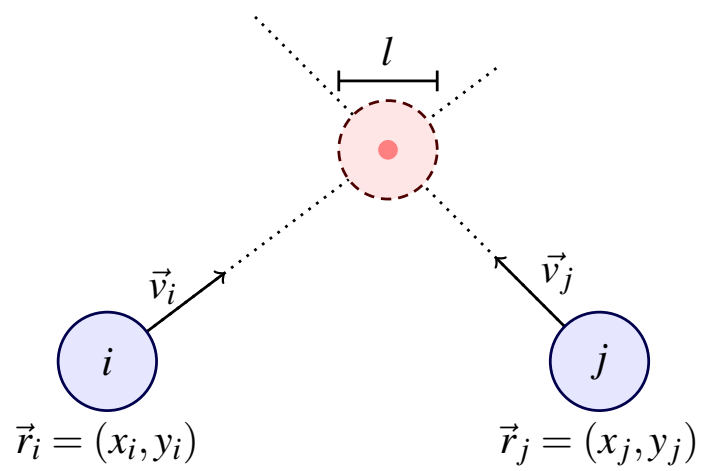

Figure 1 Schematic illustration of two colliding pedestrians. The position vectors $\vec{r}_{i}$ and $\vec{r}_{j}$ point to the centers of the disks.

(a) $\vec{v}_{i}=$ const. and $\vec{v}_{j}=0$

(b) $\vec{v}_{i}=$ const . and $\vec{v}_{j}=$ const .

These approximations simply correspond to different Taylor expansions and could be carried on to higher orders. The following considerations are restricted to these approximations and one dimension.

Assume a one-dimensional chain of $N$ pedestrians on a ring of length $L$. We denote $\left(x_{i}\right)$ the pedestrian positions and $\left(v_{i}\right)$ their velocities sorted in ascending order in the direction of motion, i.e. pedestrian $i+1$ is the predecessor of pedestrian $i$. Approximation (a) results in

$$
\tau_{1}^{i, i+1}(t)= \begin{cases}\frac{\Delta x_{i}-l}{v_{i}} & \text { for } v_{i}>0 \\ \infty & \text { else, }\end{cases}
$$

where $\Delta x_{i}=x_{i+1}-x_{i}$ is the spatial distance between centers (by taking $\Delta x_{i}=L+x_{i+1}-x_{i}$, when, due to the periodic conditions, $x_{i+1}<x_{i}$ ). If the velocity of pedestrian $i+1$ is assumed to be constant, i.e. approximation (b), the time-to-collision is

$$
\tau_{2}^{i, i+1}(t)= \begin{cases}\frac{\Delta x_{i}-l}{v_{i}-v_{i+1}} & \text { for } v_{i}>v_{i+1} \\ \infty & \text { else. }\end{cases}
$$

Both of these times are referred to as the time-to-collision. However, $\tau_{1}^{i, i+1}$ is usually denoted as the time gap. While it can be helpful to think of the time-to-collision as a time distance, it should be emphasized that it is not a metric in the mathematical sense. The TTC neither obeys the identity of indiscernibles nor the triangle inequality. It is not symmetric for the first approximation, i.e. $\tau_{1}^{i j} \neq \tau_{1}^{j i}$.

\section{Models}

Based on these approximations models can be defined by assuming that the pedestrians choose their velocity such that the time-to-collision is larger or equal than a desired time gap $T$. This gives rise to two types of models. 
The Optimal-Velocity Models: The time gap $\tau_{1}^{i j}$ can be combined with the desired time gap $T$ by requiring $\tau_{1}^{i j} \geq T$. The resulting model is

$$
v_{i}=\frac{\Delta x_{i}-l}{T},
$$

which is the first-order Optimal-Velocity model [11]. When a reaction-time $t_{R}$ is included, this gives rise to either a delayed first-order model [12] or the second-order OptimalVelocity model [4].

Time-to-Collision Models: By using the time-to-collision $\tau_{2}^{i j}$ and requiring $\tau_{2}^{i j} \geq T$, the first-order Time-to-Collision model is defined by

$$
v_{i}=v_{i+1}+\frac{\Delta x_{i}-l}{T} .
$$

If a homogeneous population of $N$ agents on a ring of length $L$ is considered, the model is generically inconsistent. An analytical solution can be obtained easily but requires $N \cdot l=L$. Therefore, only trivial solutions with

$$
\Delta x_{i}=l \quad \text { and } \quad v_{i}=c \geq 0
$$

exist. The solution (7) can be understood intuitively from the definition of the model: Since, according to (6), every agent wants to move faster or equally fast as his or her predecessor, all need to have the same velocity and a zero headway.

A maximal velocity $v_{\max }$ can be added to (6) as

$$
v_{i}=\min \left(v_{\max }, v_{i+1}+\frac{\Delta x_{i}-l}{T}\right) .
$$

In this model a solution exists for all densities, i.e.

$$
\Delta x_{i} \geq l \quad \text { and } \quad v_{i}=v_{\max } .
$$

A second-order model with a reaction time $t_{R}$ can be defined as

$$
\dot{v}_{i}=\frac{1}{t_{R}}\left(v_{i+1}+\frac{\Delta x_{i}-l}{T}-v_{i}\right)
$$

which looks similar to the Full-Velocity-Difference model [13]. However, in the FVDM the velocity relaxes to $\left(\Delta x_{i}-l\right) / T$ to which $\Delta v$ is added. In (10) the velocity relaxes to $v_{i+1}+\left(\Delta x_{i}-l\right) / T$. Consequently, the only homogeneous solutions are the solutions of the first-order model (6) described above.

In a simplified picture, one-dimensional traffic consists of a homogeneous free-flow for small densities and a congested, heterogeneous phase in which stop-and-go dynamics are visible. In continuous models, this is typically reproduced by deterministic instabilities of the homogeneous solution. The homogeneous solutions of (10) are, according to the conditions derived in [14], always linearly unstable. 
Interim Conclusion: The two approximations correspond to extreme cases. In the Optimal Velocity model (5), from the time-to-collision point of view, all pedestrians assume that the preceding pedestrian will stop at any time. The relative velocities are not considered and the model only depends on the spatial distances. In the time-to-collision model (6), based on $\tau_{2}^{i j}$, the pedestrians assume that the others never stop or go slower but continue to move with their current velocity. This results either in (stationary) solutions only for $\rho=\rho_{\max }=1 / l$, i.e. (7), or a very high flow for large densities as it can be seen from (9).

The approximations used have a natural interpretation as different types of anticipation used by the pedestrians. Since both approximations correspond to bad or unrealistic anticipation strategies, one should look for better strategies to estimate the time-to-collision. Or, more precisely, how do pedestrians approximate $v_{i+1}$ in the time interval $\left[t, t+\tau^{i j}\right]$ ? This is not trivial to model since $\tau^{i j}$ depends on $v_{i+1}$ as well.

This approach gives rise to a class of models, which are all based on the principle that the pedestrians choose their velocity such that the time-to-collision is larger or equal than a desired time gap $T$ and only differ in the way the TTC is estimated.

\section{Anticipation Strategies}

In this section different anticipation strategies, i.e. ways to estimate $v_{i+1}$, are proposed. The approximations of $v_{i+1}$ are denoted as $v_{i+1}^{\text {approx }}$. The corresponding first- and secondorder models are

$$
v_{i}=v_{i+1}^{\text {approx }}+\frac{\Delta x_{i}-l}{T}
$$

and

$$
\dot{v}_{i}=\frac{1}{t_{R}}\left(v_{i+1}^{\text {approx }}+\frac{\Delta x_{i}-l}{T}-v_{i}\right) .
$$

The stability conditions of the homogeneous solutions apply to the second-order models (12) only. The homogeneous solutions of the first-order models (11) are always linearly stable.

Trust Parameter: One might introduce a new parameter $\alpha \in[0,1]$ such that

$$
v_{i+1}^{\text {approx }}=\alpha v_{i+1} .
$$

The parameter $\alpha$ can be interpreted as the degree of trust in the current velocity of the preceding pedestrian. A small $\alpha$ corresponds to estimating a strong tendency of the preceding agent to slow down. While for $\alpha$ close to one, the pedestrians assume that the others tend to keep on moving with their current velocity. For $\alpha=0$, the OV model is obtained and for $\alpha=1$ the TTC model. In general, the parameter could depend on various other quantities, e.g. the normalized global density in the form $\alpha=1-\rho / \rho_{\max }$.

Reasonable (stationary) solutions only exist for $\alpha<1$. Hence, this approach does not allow anticipating an increasing velocity of the preceding pedestrian. The homogeneous 
solution is linearly stable for

$$
t_{R}<T \frac{\left(1-\alpha^{2}\right)}{2}
$$

which was derived from [14]. The model becomes more unstable as the trust $\alpha$ increases. While a larger $\alpha$ allows a higher flow, it destabilizes the system.

Inverse Density: The inverse density is a measure of the available space for each pedestrian. As for the global density, $\rho^{-1}=L / N$ corresponds to the mean headway $\langle\Delta x\rangle$. The velocity might be approximated by combining it with the linear Optimal-Velocity function from $(5)^{1}$

$$
v_{i+1}^{\text {approx }}=\frac{\langle\Delta x\rangle-l}{T}=\frac{\rho^{-1}-l}{T} .
$$

Thus, a density-dependent, constant term is added to the desired velocity. The homogeneous solution has twice the velocity than the one of the OV model. However, the stability condition

$$
t_{R}<\frac{1}{2} T
$$

obtained from [14] is the same as the one of the second-order OV model [4]. The velocity can be increased while sustaining the stability of the model.

The Voronoi density is a good measure of the local density [15]. It corresponds to the inverse of the space occupied by one pedestrian. In one dimension the space of pedestrian $i+1$ is the difference between the midpoints of pedestrians $(i, i+1)$ and $(i+1, i+2)$. The approximated velocity reads

$$
v_{i+1}^{\text {approx }}=\frac{\frac{1}{2}\left(x_{i+2}-x_{i}\right)-l}{T} .
$$

Hence, an interaction with the next agent in front is included in the model. The homogeneous solution of the second-order model is, according to [16], linearly stable for

$$
t_{R}<\frac{3}{8} T
$$

The homogeneous solution has the same velocity as the global density approximation but the stability conditions differ.

Alternatively, the own headway might be used to approximate the velocity of the next pedestrian, i.e.

$$
v_{i+1}^{\text {approx }}=\frac{\Delta x_{i}-l}{T} .
$$

This assumes that the pedestrian $i+1$ is simply in the same situation as $i$. The corresponding stability condition reads

$$
t_{R}<\frac{1}{4} T
$$

For $T \rightarrow 2 T$, the corresponding second-order model (12) has the same stability condition and homogeneous velocity as the second-order OV model from [4].

\footnotetext{
${ }^{1}$ This can be interpreted as the following: Pedestrian $i$ assumes that $j$ determines his or her velocity using the first approximation in which $j$ assumes that all other pedestrians might stop at any time.
} 


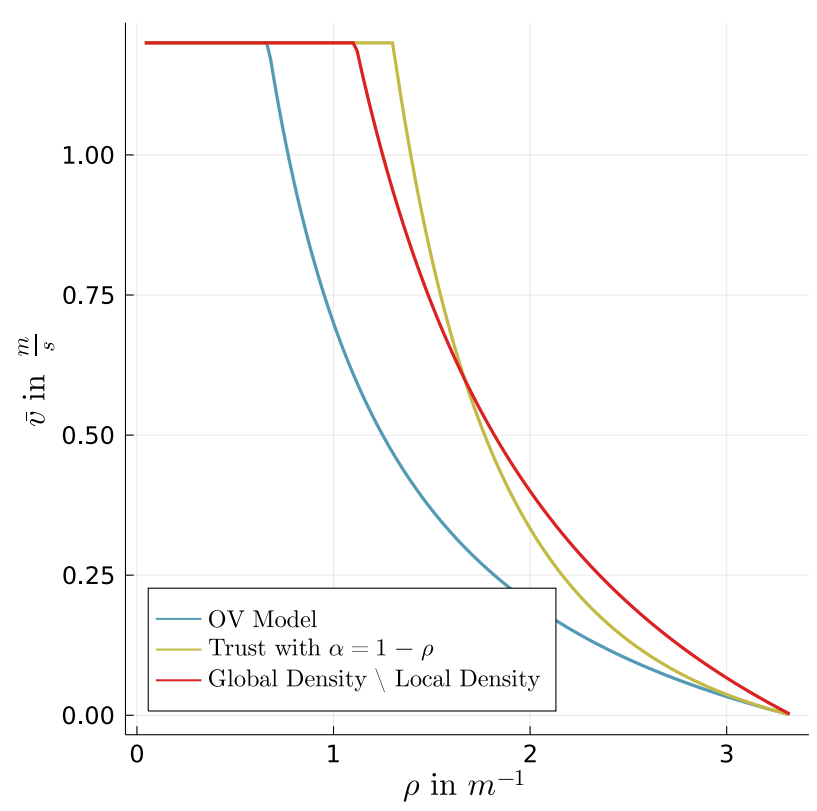

Figure 2 The global fundamental diagrams of the first-order OV model (5) and the first-order models (11) based on the density dependent anticipations using the local density (17), the global density (15) and (13) with $\alpha=1-\rho / \rho_{\max }$. The parameters are $v_{\max }=1.2 \frac{\mathrm{m}}{\mathrm{s}}, T=1 \mathrm{~s}$ and $l=0.3 \mathrm{~m}$. The approximations using the local and the global density lead to the same fundamental diagram. The values were obtained using simulations of the models.

\section{Numerical Results}

The simulations were carried out using periodic boundary conditions and an explicit Euler-Scheme with $d t=0.01 \mathrm{~s}$. Typical values for the parameters were used, i.e. $T=1 \mathrm{~s}$, $v_{\max }=1.2 \frac{\mathrm{m}}{\mathrm{s}}$ and $l=0.3 \mathrm{~m}$.

In Fig. 2 fundamental diagrams for different proposed first-order models are shown. All models have a similar fundamental diagram that resembles the shape observed in experiments, e.g. [17]. The approximations using the local and the global density, i.e. (17) and (15) have the same fundamental diagram. The OV model (5) has the smallest flow for all densities. If the time gap in the OV model is half of the one in the global or local density approximations, they result in exactly the same fundamental diagram. Compared to the global/local density approximation, $\alpha=1-\rho$ has a higher flow for low densities. In the highly congested system, it is vice versa.

In Fig. 3, the influence of the trust parameter $\alpha$, i.e. (13), on the fundamental diagram is shown. The expected $1 / \rho$-shape is maintained if $\alpha$ is small. However for $\alpha$ close to 1 , the pedestrians move with $v_{\max }$ even for very large densities.

\section{Conclusion}

In this paper a general definition of the time-to-collision was given (2). Typical approximations were applied to calculate it in a one-dimensional setting, i.e. (3) and (4). By 


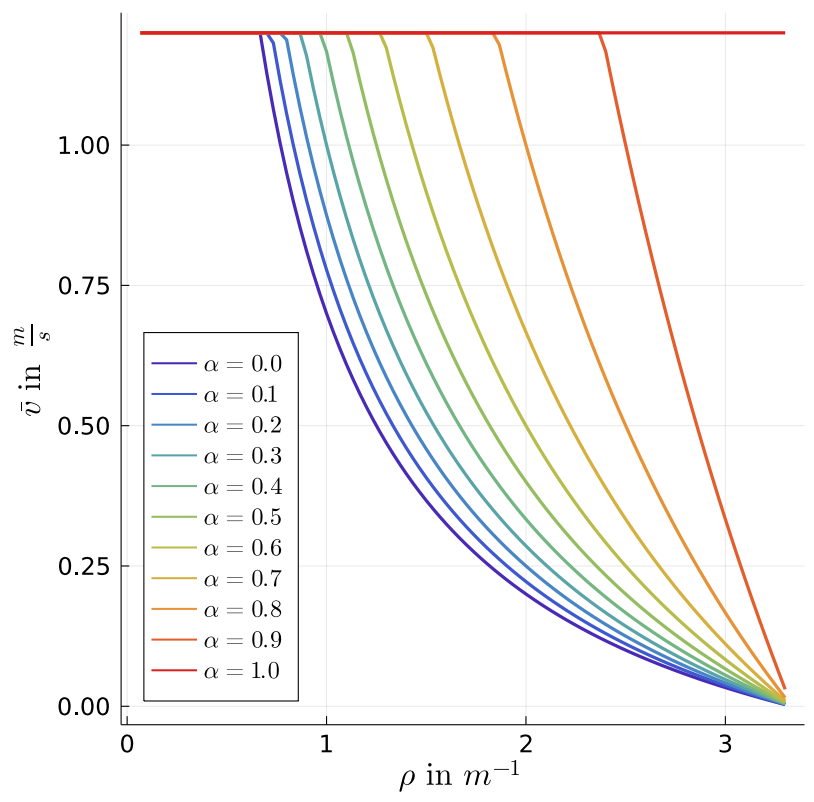

Figure 3 The global fundamental diagram for the proposed first-order model (11) based on the anticipation scheme (13) with a constant trust-parameter $\alpha$. The parameters are $v_{\max }=1.2 \frac{\mathrm{m}}{\mathrm{s}}, T=1 \mathrm{~s}$ and $l=0.3 m$. The trust-parameter $\alpha$ is varied from $\alpha=0$, i.e. the OV model to $\alpha=1$, i.e. the TTC model. The values were obtained using simulations of the models.

combining the TTC with a desired time gap several models were defined.

The first-order OV model (5) can be obtained using this approach. From this perspective, the OV model does inhibit anticipation. The pedestrians assume that all other pedestrians stop while he or she keeps on moving with a constant velocity. In some situations this might be a reasonable assumption. But, in general, it probably does not reflect the anticipation strategy of pedestrians.

The time-to-collision can be approximated by assuming that all velocities are constant, i.e. (4). This leads to the TTC model (6) which is generically inconsistent for periodic boundaries and a homogeneous crowd. The corresponding analytical solutions can easily be obtained but the solution space, i.e. (7) and (9), is very restricted. Furthermore the model exhibits very large flows at high densities. This unrealistic behavior is related to the used approximations which should be interpreted as an anticipation strategy of the agents.

Therefore, several explicit anticipation strategies were proposed. The corresponding models show reasonable dynamics and fundamental diagrams. The stability conditions for the inverse density approximations are $t_{R}<4 / 8 T$ for the global density (15), $t_{R}<3 / 8 T$ for the Voronoi density of the next pedestrian (17), and $t_{R}<2 / 8 T$ if the headway of pedestrian $i$ is used (19). While the stability conditions are different the inverse density approximations all lead to the same velocity in the homogeneous solution, i.e. $v=2\left(\rho^{-1}-l\right) / T$. This is twice the homogeneous velocity of the simple OV model (5).

In conclusion, single-file pedestrian models can be defined using the time-to-collision. However, one needs to be aware of the (implicit) anticipation strategies that are used in 
approximations. If the proposed estimates of the TTC resemble the empirical results from [6], i.e. are able to parametrize their pair distribution, is unclear. Furthermore, the pair distribution of the proposed models could be examined and compared to the empirical results. These questions, however, lie beyond the scope of this contribution and could be addressed in future studies.

The proposed ansatz can be used for a two-dimensional model as well. Since the timeto-collision becomes non-trivial and non-linear in two-dimensions this could give rise to an interesting model. It should exhibit many crucial mechanisms while using few assumptions.

Acknowledgements JC, MC and AT acknowledge the Franco-German research project MADRAS funded in France by the Agence Nationale de la Recherche (ANR, French National Research Agency), grant number ANR-20-CE92-0033, and in Germany by the Deutsche Forschungsgemeinschaft (DFG, German Research Foundation), grant number 446168800.

\section{References}

[1] Nicolas, A., Kuperman, M., Ibañez, S., Bouzat, S., Appert-Rolland, C.: Mechanical response of dense pedestrian crowds to the crossing of intruders. Scientific Reports 9(1), 105 (2019). doi:10.1038/s41598-018-36711-7

[2] Helbing, D., Molnár, P.: Social force model for pedestrian dynamics. Phys. Rev. E 51, 4282-4286 (1995). doi:10.1103/PhysRevE.51.4282

[3] Burstedde, C., Klauck, K., Schadschneider, A., Zittartz, J.: Simulation of pedestrian dynamics using a two-dimensional cellular automaton. Physica A 295, 507-525 (2001). doi:10.1016/S0378-4371(01)00141-8

[4] Bando, M., Hasebe, K., Nakayama, A., Shibata, A., Sugiyama, Y.: Dynamical model of traffic congestion and numerical simulation. Phys. Rev. E 51(2), 10351042 (1995). doi:10.1103/PhysRevE.51.1035

[5] Nakayama, A., Hasebe, K., Sugiyama, Y.: Instability of pedestrian flow and phase structure in a two-dimensional optimal velocity model. Physical Review E 71(3), 036121 (2005). doi:10.1103/PhysRevE.71.036121

[6] Karamouzas, I., Skinner, B., Guy, S.J.: A universal power law governing pedestrian interactions. Phys. Rev. Lett. 113(5), 238701 (2014). doi:10.1103/PhysRevLett.113.238701

[7] Moussaïd, M., Helbing, D., Theraulaz, G.: How simple rules determine pedestrian behavior and crowd disasters. PNAS 108(17), 6884-6888 (2011). doi:10.1073/pnas.1016507108 
[8] Xiao, Y., Gao, Z., Qu, Y., Li, X.: A pedestrian flow model considering the impact of local density: Voronoi diagram based heuristics approach. Transportation Research Part C: Emerging Technologies 68, 566-580 (2016). doi:10.1016/j.trc.2016.05.012

[9] Asano, M., Iryo, T., Kuwahara, M.: Microscopic pedestrian simulation model combined with a tactical model for route choice behaviour. Transport Res. C-Emer. 18(6), 842-855 (2010). doi:10.1016/j.trc.2010.01.005

[10] von Krüchten, C.: Development of a cognitive and decision-based model for pedestrian dynamics. Ph.D. thesis, Universität zu Köln, Köln (2019)

[11] Pipes, L.A.: An operational analysis of traffic dynamics. J. Appl. Phys. 24(3), 274 - 281 (1953). doi:10.1063/1.1721265

[12] Newell, G.F.: Nonlinear Effects in the Dynamics of Car Following. Operations Research 9(2), 209-229 (1961). doi:10.1287/opre.9.2.209

[13] Jiang, R., Wu, Q., Zhu, Z.: Full velocity difference model for a car-following theory. Physical Review E 64(1), 017101 (2001). doi:10.1103/PhysRevE.64.017101

[14] Lassarre, S., Roussignol, M., Tordeux, A.: Linear stability analysis of first-order delayed car-following models on a ring. Physical Review E 86, 036207 (2012). doi:10.1103/PhysRevE.86.036207

[15] Steffen, B., Seyfried, A.: Methods for measuring pedestrian density, flow, speed and direction with minimal scatter. Physica A 389(9), 1902-1910 (2010). doi:10.1016/j.physa.2009.12.015

[16] Tordeux, A., Chraibi, M., Schadschneider, A., Seyfried, A.: Influence of the number of predecessors in interaction within acceleration-based flow models. Journal of Physics A: Mathematical and Theoretical 50(34), 345102 (2017). doi:10.1088/1751-8121/aa7fca

[17] Seyfried, A., Portz, A., Schadschneider, A.: Phase coexistence in congested states of pedestrian dynamics. In: Bandini, S., Manzoni, S., Umeo, H., Vizzari, G. (eds.) Cellular Automata, Lecture Notes in Computer Science, vol. 6350, pp. 496-505. Springer-Verlag Berlin Heidelberg (2010). doi:10.1007/978-3-642-15979-4_53 